\title{
Influência da composição granulométrica das partículas de resíduos de madeira nas propriedades de compósitos minerais: parte 1 - cimento-madeira
}

Influence of particle size of wood waste on the properties of mineral composites: part 1 - cement-wood

\section{Thaísa Mariana Santiago Rocha \\ Leonardo Fagundes Rosemback Miranda Carlos Frederico Alice Parchen}

\section{Resumo}

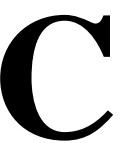

ompósitos de fibras naturais são uma alternativa para absorver os resíduos de construção e demolição (RCD) de madeira, o que reduz os impactos ambientais e contribui com o desenvolvimento de materiais, produtos e processos mais sustentáveis. Pesquisadores têm obtido resultados promissores com compósitos contendo diferentes dimensões de partículas. O objetivo deste estudo foi avaliar a influência da composição granulométrica das partículas de madeira provenientes de RCD nas propriedades físicas e mecânicas de compósitos de cimento-madeira. As partículas de madeira foram moídas em moinho de martelos e caracterizadas. Foi utilizado o cimento Portland, o aditivo acelerador de pega e a água. Os compósitos foram produzidos com diferentes relações aglomerante-madeira com relação água-cimento fixada em 0,50 em massa. A partir dos resultados dos ensaios no estado fresco e endurecido, verificou-se que os valores de massa unitária das partículas de madeira influenciaram nas propriedades físicas e, consequentemente, no desempenho mecânico dos compósitos. Como conclusão tem-se que a composição granulométrica P1,2 resultou na menor densidade de massa endurecida, enquanto a composição granulométrica P1 proporcionou maior resistência à tração na flexão.

Palavras-chave: Resíduos de Construção e Demolição (RCD). Compósitos. Partículas de madeira. Composição granulométrica. Cimento Portland.

\begin{abstract}
Natural fibre composites are an alternative to absorb wood construction and demolition waste $(C D W)$, reducing environmental impact, contributing to the development of more sustainable materials, products, and processes.

Researchers have obtained promising results with composites containing different particle sizes. The aim of this study was to evaluate the influence of particle size distribution of wood from CDW on the physical and mechanical properties of cement-wood composites. The wood particles were milled in hammer mills and characterised. Portland cement, a setting time accelerator and water were used. The composites were produced with different binder/wood ratios with the water/cement ratio fixed at 0.50 (by mass). The results of the tests in the fresh and hardened state showed that the values of unit weight of the wood particles influenced the physical properties and, consequently, the mechanical performance of the composites. The study concluded that the P1,2 particle size distribution resulted in lower hardened mass density, while the $P 1$ particle size distribution provided higher tensile strength in flexion.
\end{abstract}

${ }^{3}$ Carlos Frederico Alice Parchen ${ }^{3}$ Universidade Federal do Paraná Curitiba - PR - Brasil

Recebido em 29/03/18 Aceito em 12/11/18
Keywords: Construction and Demolition Waste (CDW). Composites. Wood particles. Particle size distribution. Portland cement.

ROCHA, T. M. S.; MIRANDA, L. F. R.; PARCHEN, C. F. A. Influência da composição granulométrica das partículas de 


\section{Introdução}

A indústria da construção civil consome grande quantidade de energia, além de provocar diversos impactos ao ambiente, como emissão de gases do efeito estufa, consumo de água e geração de resíduos sólidos (ASHOUR et al., 2015). Um volume considerável dos resíduos de construção e demolição (RCD) é gerado por essa indústria, o que pode representar algo entre $13 \%$ e $40 \%$ do total de resíduos sólidos gerados (HUANG et al., 2002; YUAN; SHEN, 2011). A geração de RCD de madeira está diretamente relacionada ao processo construtivo realizado em determinada região (PEDREÑO-ROJAS et al., 2017), podendo atingir, aproximadamente, $23 \%$ na Suécia, $10 \%$ na Bélgica e 4\% na Alemanha do total de resíduo produzido (EUROPEAN..., 2017).

Os compósitos de fibras naturais podem ser uma alternativa, principalmente em países em desenvolvimento, sendo capazes de absorver os resíduos, de forma a reduzir os impactos ambientais (KHORAMI; GANJIAN, 2013), além de contribuir com o desenvolvimento de materiais, produtos e processos mais sustentáveis (AKIL et al., 2011; VÄNTSI; KÄRKI, 2015). Ademais, as fibras naturais têm um excelente potencial para reduzir as emissões de $\mathrm{CO}_{2}$ e diminuir o consumo de recursos não renováveis (PERVAIZ; SAIN, 2003), podendo proporcionar benefícios ambientais quando comparadas à utilização de fibras poliméricas, confirmados através de análises de ciclo de vida (PIETRINI et al., 2007; XU et al., 2008; MILLER; LEPECH; BILLINGTON, 2013), além de apresentar viabilidade técnica e econômica (SAVASTANO JUNIOR et al., 1999).

Materiais compósitos podem ser definidos como misturas heterogêneas de duas ou mais fases que são utilizados para produzir um novo material com propriedades superiores às de seus constituintes (KELLY, 1994; HULL; CLYNE, 1996). Os compósitos minerais associados a fibras lignocelulósicas geralmente são produzidos a partir da mistura de um aglomerante mineral com as partículas de madeira. Entre os aglomerantes utilizados, os principais são o gesso, o cimento de magnésio e o cimento Portland (YOUNGQUIST, 1999). A utilização da madeira em matrizes cimentícias pode contribuir para a diminuição da densidade, o aumento da resistência à flexão (SAVASTANO JUNIOR et al., 2009; KHORAMI; GANJIAN, 2013; SELLAMI; MERZOUD; AMZIANE, 2013), o controle da iniciação e da propagação de microfissuras (TOLEDO FILHO et al., 2005; TONOLI et al., 2013) e a melhoria da resistência ao impacto (RAMAKRISHNA; SUNDARARAJAN, 2005) e das propriedades térmicas (CORINADELSI; MAZZOLI; SIDDIQUE, 2016). Compósitos que contêm fibras geralmente são macios e dúcteis em torno das fibras - que são geralmente mais fortes -, mas podem ser frágeis em relação à matriz (SMITH, 1994). De acordo com a regra das misturas, cada fase constituinte do compósito contribui para as propriedades efetivas do compósito, sendo essa função dependente apenas da fração volumétrica dos constituintes. Entretanto, essa teoria não considera a presença de vazios nem os efeitos da interface entre a matriz e a carga.

Compósitos produzidos com diferentes dimensões de partículas de madeira têm apresentado resultados promissores (BOUGUERRA et al., 2002; MATHUR, 2006; ADEDEJI; AJAYI, 2008), tornando-se uma opção leve e econômica quando comparados às fibras de vidro, por exemplo.

A primeira parte do trabalho objetiva avaliar a influência da composição granulométrica das partículas de madeira provenientes de resíduos de construção e demolição (RCD) nas propriedades físicas e mecânicas de compósitos de cimentomadeira (Parte 1). A segunda parte, objeto de publicação posterior, refere-se às análises realizadas em compósitos de gesso-madeira (Parte 2).

\section{Programa experimental}

Foram produzidos compósitos de cimento-madeira cujas partículas de madeira são provenientes de resíduos de construção e demolição (RCD). Os resíduos de compensado, eucalipto e pínus foram moídos e classificados quanto a sua composição granulométrica. Os compósitos produzidos foram avaliados com relação às propriedades no estado fresco e no estado endurecido aos 28 dias.

\section{Materiais}

Foram utilizados o cimento Portland (CP V ARI) (Tabela 1) - com finura blaine de $4.955 \mathrm{~cm}^{2} / \mathrm{g} \mathrm{e}$ massa específica de $2,98 \mathrm{~g} / \mathrm{cm}^{3}$ - e o aditivo acelerador de pega à base de silicato de sódio (no teor de 5\% com relação à massa do cimento), cujas características podem ser observadas na Tabela 2.

Foram obtidos três tipos diferentes de madeira provenientes de resíduos de construção e demolição (RCD): compensado, eucalipto e pínus (Figura 1). Os resíduos de madeira foram coletados em canteiros de obras situados nos estados do Paraná e de Santa Catarina. Estes foram moídos em um moinho de martelos portátil com potência de 5,0 cv e 1.160 RPM. 
O procedimento de moagem realizado foi o mesmo para todas as composições granulométricas apresentadas e classificadas neste estudo (Quadro 1). O tempo que o resíduo levou para percorrer o moinho de martelos e originar as partículas foi de 3 s. Entretanto, as composições granulométricas identificadas como P1 foram submetidas a apenas uma repetição do procedimento de moagem. As composições P3 foram moídas por três vezes consecutivas. Por fim, as composições granulométrcias identificadas como P1,2 referem- se às partículas moídas e obtidas a partir do passante na peneira $1,2 \mathrm{~mm}$.

A identificação e a classificação das diferentes composições granulométricas das partículas de madeira foram realizadas no intuito de viabilizar a verificação da influência destas nos compósitos cimentícios. A repetição da moagem foi realizada para que fosse possível obter o volume necessário de cada granulometria das partículas de madeira para a realização dos ensaios.

\section{Tabela 1 - Composição química do cimento Portland}

\begin{tabular}{c|c|c|c}
\hline \multicolumn{4}{c}{ Composição química } \\
\hline Perda ao fogo (\%) & MgO (\%) & SO $_{3}$ (\%) & Resíduo insolúvel (\%) \\
\hline 3,5 & 5,5 & 3,1 & 11,8 \\
\hline
\end{tabular}

Tabela 2 - Características do aditivo acelerador de pega

\begin{tabular}{|c|c|c|c|c|c|}
\hline Identificação & Densidade $\left(\mathrm{g} / \mathrm{cm}^{3}\right)$ & Estado físico & Cor & Composição química & Dosagem (\%) \\
\hline Aditivo & 1,27 & Líquido & Incolor & Silicato de sódio & 3,0 a 15,0 \\
\hline
\end{tabular}

\section{Figura 1 - Preparo, coleta e moagem dos resíduos de construção e demolição (RCD) de madeira}

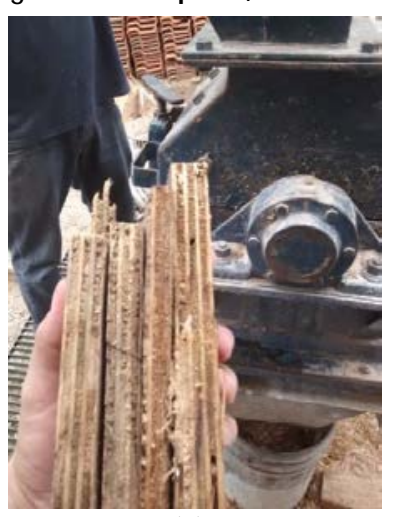

(a)

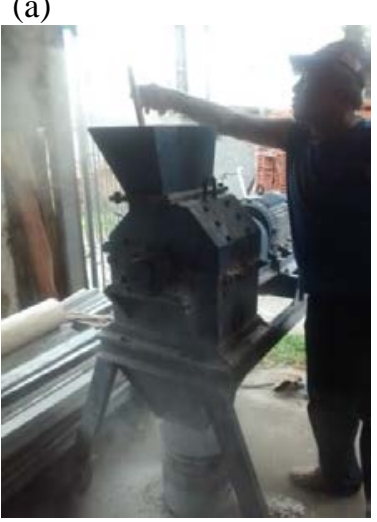

(d)

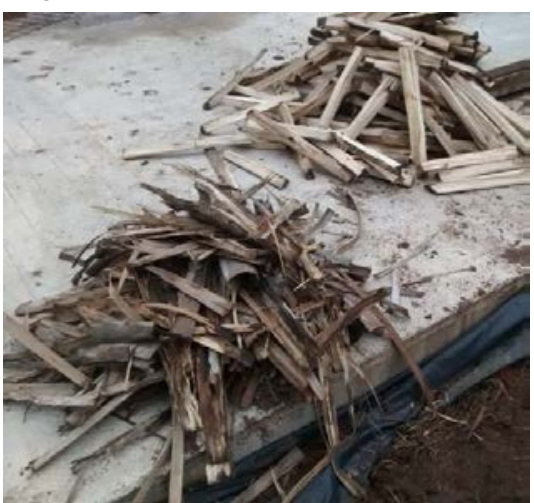

(b)

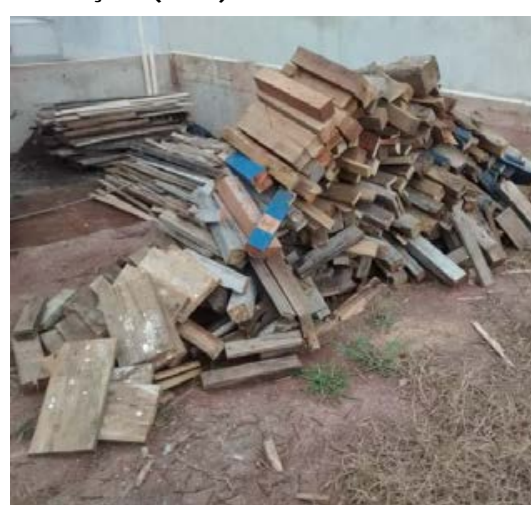

(c)

Fonte: Rocha (2017).

Nota: Legenda:
[a] RCD de compensado;
[b] RCD de eucalipto descascado;
[c] RCD de pínus;
[d] moinho de martelos utilizado para a moagem do RCD; e
[e] partículas de madeira provenientes de RCD. 
Quadro 1 - Identificação das partículas de madeira

\begin{tabular}{|c|l|}
\hline Identificação & \multicolumn{1}{c|}{ Características } \\
\hline P1 & Partículas geradas após a moagem realizada uma vez (1x) no moinho \\
\hline P3 & Partículas geradas após a moagem realizada três vezes (3x) no moinho \\
\hline P1,2 & Partículas geradas a partir do passante na peneira 1,2 mm \\
\hline
\end{tabular}

\section{Métodos}

O pré-tratamento das partículas de madeira foi realizado em água fria por 48 h numa proporção de 1:10 (madeira:água). Em seguida, as partículas foram secas ao ar por $72 \mathrm{~h}$ para que então fosse realizada a secagem em estufa a $80^{\circ} \mathrm{C}$ por $24 \mathrm{~h}$. Para a produção dos compósitos, a relação água-cimento foi fixada em 0,50 (em massa) (PARCHEN, 2012). Como as partículas encontravam-se secas, foi aplicada a compensação de água proposta por Simatupang (1979) (Equação 1).

$C a=R_{a / a} \times C+(P S F-U) \times M$

Eq. 1

Onde:

Ca é o consumo de água (g);

$R_{a / a}$ é a relação água-aglomerante;

$C$ é o consumo de aglomerante (g);

$P S F$ é o ponto de saturação das fibras (adotado como 30\%);

$U$ é o teor de umidade da madeira (\%); e

$M$ é a massa de madeira (g).

\section{Análise comparativa das composições granulométricas das partículas de madeira}

Para comparar as composições granulométricas das partículas obtidas após a moagem, foram realizados os ensaios de caracterização para a determinação da massa unitária C29 (AMERICAN..., 2017), da composição granulométrica C136 (AMERICAN..., 2014a) e do teor de umidade D4442 (AMERICAN..., 2016). O teor de umidade das partículas de madeira foi utilizado para determinar a quantidade de água adicionada na mistura, conforme descrito na Equação 1.

\section{Análise da influência das composições granulométricas das partículas no estado fresco}

Para avaliar a influência das composições granulométricas das partículas no estado fresco foram produzidos compósitos (Tabela 3) e realizados os ensaios de determinação do tempo de pega C308 (AMERICAN..., 2012), do índice de consistência C1437 (AMERICAN..., 2015) e de densidade de massa fresca C270 (AMERICAN..., 2014d). A proporção aglomerante:madeira foi de 1:0,075 (em massa), conforme proposto por Moslemi, Garcia e Hofstrand (1983), Latorraca, Iwakiri e Lelis (1999), Latorraca (2000), Lima (2009) e Lima e Iwakiri (2011). Além disso, foram produzidas amostras de referência ( $0 \%$ de partículas de madeira).

\section{Análise da influência das composições granulométricas das partículas no estado endurecido}

Para avaliar a influência das composições granulométricas das partículas no estado endurecido foram produzidos corpos de provas prismáticos (4x4x16 cm³) (Tabela 4).

Os corpos de prova foram curados em câmara seca (a $21{ }^{\circ} \mathrm{C} \pm 2{ }^{\circ} \mathrm{C}$ e com umidade inferior a $50 \%$ ) e ensaiados, aos 28 dias, quanto à densidade de massa endurecida, à resistência à tração na flexão C348 (AMERICAN..., 2014b) e à compressão C349 (AMERICAN..., 2014c).

Nessa etapa, além da proporção aglomerantemadeira de $7,5 \%$ e a amostra de referência ( $0 \%$ de partículas de madeira), foram produzidos compósitos nas proporções 11\% (1:0,11) e 15\% $(1: 0,15)$ no intuito de avaliar também a influência dos maiores teores de partículas de madeira nas propriedades mecânicas dos compósitos. Não foram realizados os ensaios no estado fresco para as proporções aglomerante-madeira de $11 \%$ e de $15 \%$. Isso porque a quantidade de partículas na mistura impediu a execução adequada do ensaio de tempo de início e de fim de pega, mascarando os resultados. Além disso, espera-se que uma maior quantidade de mistura na composição contribua para a diminuição do índice de consistência e da densidade de massa endurecida.

O delineamento estatístico utilizado foi análise fatorial com ênfase na natureza dos tratamentos, sendo estes a dimensão das partículas (P1, P3 e $\mathrm{P} 1,2$ ), o tipo de partícula (compensado, eucalipto e pínus) e a proporção de madeira na composição (7,5\%, 11\% e 15\%). Para a comparação entre as médias, utilizou-se ANOVA em conjunto com o teste de Tukey, no nível de probabilidade de 95\%. 
Tabela 3 - Compósitos de madeira produzidos para avaliação no estado fresco

\begin{tabular}{|c|c|c|c|c|}
\hline \multicolumn{3}{|c|}{ Identificação } & $\begin{array}{c}\text { Relação água- } \\
\text { aglomerante (em massa) }\end{array}$ & $\begin{array}{c}\text { Proporção aglomerante-madeira } \\
\text { (em massa) }\end{array}$ \\
\hline \multirow{10}{*}{ 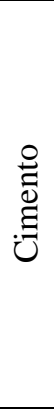 } & \multirow{3}{*}{$\mathrm{P} 1$} & Compensado & \multirow{9}{*}{0,50} & \multirow{9}{*}{$7,5 \%$} \\
\hline & & Eucalipto & & \\
\hline & & Pínus & & \\
\hline & \multirow{3}{*}{ P3 } & Compensado & & \\
\hline & & Eucalipto & & \\
\hline & & Pínus & & \\
\hline & \multirow{3}{*}{$\mathrm{P} 1,2$} & Compensado & & \\
\hline & & Eucalipto & & \\
\hline & & Pínus & & \\
\hline & & Ref & 0,50 & $0 \%$ \\
\hline
\end{tabular}

Tabela 4 - Compósitos de madeira produzidos para avaliação no estado endurecido

\begin{tabular}{|c|c|c|c|c|}
\hline \multicolumn{3}{|c|}{ Identificação } & $\begin{array}{l}\text { Relação água-aglomerante } \\
\text { (em massa) }\end{array}$ & $\begin{array}{c}\text { Proporção aglomerante- } \\
\text { madeira (em massa) }\end{array}$ \\
\hline \multirow{28}{*}{ 苞 } & & Compensado & \multirow{9}{*}{0,50} & \multirow{9}{*}{$7,5 \%$} \\
\hline & $\mathrm{P} 1$ & Eucalipto & & \\
\hline & & Pínus & & \\
\hline & \multirow{3}{*}{ P3 } & Compensado & & \\
\hline & & Eucalipto & & \\
\hline & & Pínus & & \\
\hline & \multirow{3}{*}{$\mathrm{P} 1,2$} & Compensado & & \\
\hline & & Eucalipto & & \\
\hline & & Pínus & & \\
\hline & \multirow{3}{*}{ P1 } & Compensado & \multirow{9}{*}{0,50} & \multirow{9}{*}{$11 \%$} \\
\hline & & Eucalipto & & \\
\hline & & Pínus & & \\
\hline & \multirow{3}{*}{ P3 } & Compensado & & \\
\hline & & Eucalipto & & \\
\hline & & Pínus & & \\
\hline & \multirow{3}{*}{$\mathrm{P} 1,2$} & Compensado & & \\
\hline & & Eucalipto & & \\
\hline & & Pínus & & \\
\hline & \multirow{3}{*}{ P1 } & Compensado & \multirow{9}{*}{0,50} & \multirow{9}{*}{$15 \%$} \\
\hline & & Eucalipto & & \\
\hline & & Pínus & & \\
\hline & \multirow{3}{*}{ P3 } & Compensado & & \\
\hline & & Eucalipto & & \\
\hline & & Pínus & & \\
\hline & \multirow{3}{*}{$\mathrm{P} 1,2$} & Compensado & & \\
\hline & & Eucalipto & & \\
\hline & & Pínus & & \\
\hline & & Ref & 0,50 & $0 \%$ \\
\hline
\end{tabular}

\section{Resultados e discussão}

\section{Análise comparativa das composições granulométricas das partículas de madeira}

Os resultados dos ensaios de determinação da massa unitária C29 (AMERICAN..., 2017) e do teor de umidade D4442 (AMERICAN..., 2016) estão apresentados na Tabela 5. Os resultados de massa unitária seca indicam que, para dosagens realizadas em massa, ocorre diminuição no volume de madeira presente nos traços produzidos com as partículas P3 quando comparado às partículas $\mathrm{P} 1$ e P1,2. Isso porque a massa unitária das partículas $\mathrm{P} 3$ é superior às partículas $\mathrm{P} 1$ e $\mathrm{P} 1,2$ para todos os tipos de madeira. De modo geral, comparando os três diferentes tipos de madeira, as partículas de 
compensado $\left(0,148 \mathrm{~g} / \mathrm{cm}^{3}\right.$, em média) apresentaram maior valor de massa unitária seca, seguido pelas partículas de eucalipto $\left(0,144 \mathrm{~g} / \mathrm{cm}^{3}\right.$, em média) e de pínus $\left(0,135 \mathrm{~g} / \mathrm{cm}^{3}\right.$, em média), o que indica que compósitos produzidos com compensado tendem a apresentar menor volume de partículas. É importante destacar que a quantidade de partículas de madeira presente nos compósitos é capaz de influenciar seu desempenho mecânico (TURGUT, 2007; MORALES-CONDE; RODRÍGUEZLIÑÁN; PEDREÑO-ROJAS, 2016).

A partir da composição granulométrica das partículas de madeira (Figura 2) é possível observar que a repetição do procedimento de moagem foi eficaz em contribuir para o aumento da quantidade de partículas finas de eucalipto e pínus. Entretanto, para as partículas de compensado, o mesmo efeito não foi verificado. Isso pode estar relacionado ao fato de que, como os resíduos desse tipo de madeira foram coletados em momentos diferentes, pode ter ocorrido uma variabilidade quanto à natureza dessas partículas.

Entre os tipos de madeira utilizados neste estudo, as composições que contêm a maior quantidade de partículas menores são as de eucalipto, seguidas do pínus e, por fim, de compensado. Pesquisadores (KARADE; IRLE; MAHER, 2006) indicam que as dimensões das partículas de madeira podem influenciar no desempenho no estado fresco e no estado endurecido dos compósitos, pois, quanto menor o tamanho das partículas, menor tende a ser a compatibilidade entre o cimento e a madeira. Isso pode ser explicado pelo fato de que, quanto menor a partícula, maior a área superficial e, consequentemente, maior a quantidade de extrativos liberados na composição, provocando alterações na hidratação do cimento.

Tabela 5 - Caracterização das partículas de madeira

\begin{tabular}{c|l|c|c}
\hline \multicolumn{2}{c|}{ Amostra } & Massa unitária seca $\left(\mathbf{g} / \mathbf{c m}^{3}\right)$ & Teor de umidade (\%) \\
\hline \multirow{3}{*}{ Compensado } & P1 & 0,120 & 8,84 \\
& P3 & 0,209 & 12,67 \\
& P1,2 & 0,115 & 12,95 \\
\hline \multirow{3}{*}{ Eucalipto } & P1 & 0,145 & 8,59 \\
& P3 & 0,163 & 13,53 \\
& P1,2 & 0,125 & 13,86 \\
\hline \multirow{3}{*}{ Pínus } & P1 & 0,138 & 9,14 \\
& P3 & 0,154 & 12,32 \\
& P1,2 & 0,113 & 12,40 \\
\hline
\end{tabular}

Figura 2 - Composição granulométrica das partículas de madeira

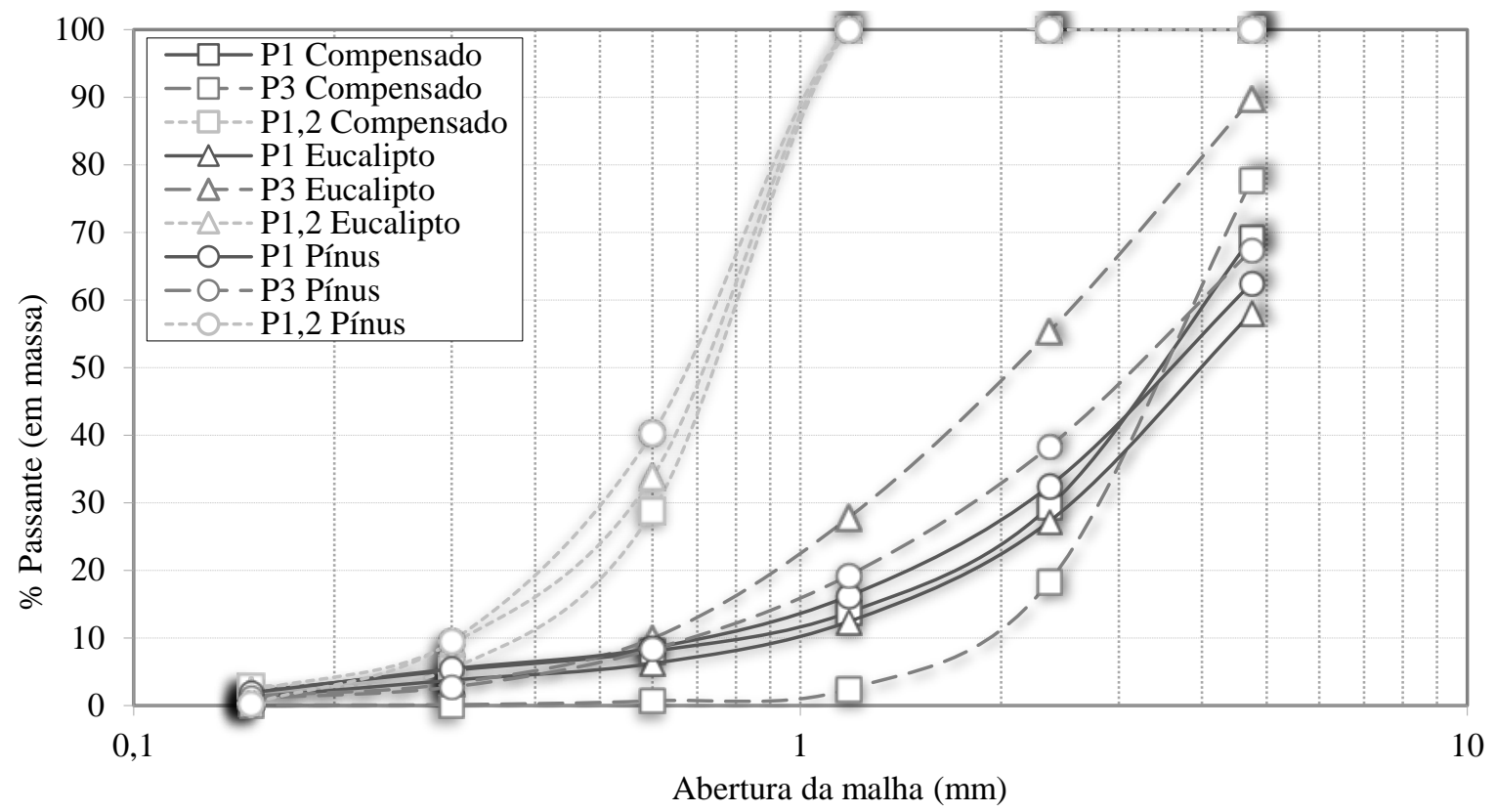




\section{Análise da influência das composições granulométricas das partículas no estado fresco}

Na Figura 3 estão apresentados os resultados obtidos a partir do ensaio de tempo de início e de fim de pega dos compósitos de cimento-madeira. É possível observar que existe uma tendência de aumento do tempo de início e fim de pega com relação aos diferentes tipos de madeira utilizados. Os compósitos produzidos com partículas de compensado apresentaram os menores valores, seguidos dos compósitos produzidos com eucalipto e pínus.

Ao observar os três diferentes tipos de madeira, verifica-se que, tanto para as partículas de compensado como para as de eucalipto, os maiores tempos de início e fim de pega foram obtidos para as partículas P3. Ou seja, para essas amostras a presença de partículas com menores dimensões $(\mathrm{P} 1,2)$ não apresentou influência. Pesquisadores indicam que cada espécie de madeira possui diferentes tipos e quantidades de extrativos de madeira, o que provoca diferentes efeitos sobre a hidratação do cimento (NA et al., 2014).

Entretanto, para os compósitos de cimento-madeira produzidos com partículas de pínus, é possível observar o efeito significativo da presença das partículas menores. Comparando os tempos de início e fim de pega entre as partículas P1 e P1,2, é possível observar um aumento de $200 \%$ e de $100 \%$ respectivamente. Ou seja, quanto menor a partícula, maior é a área superficial e, consequentemente, maior é a quantidade de extrativos livres na mistura (KARADE; IRLE; MAHER, 2006).
Ao verificar os resultados das amostras de referência, é importante destacar que o aditivo acelerador de pega utilizado mostrou-se eficiente para diminuir o tempo de início de pega dos compósitos produzidos com partículas de madeira, diferentemente do observado para o tempo de fim de pega quando comparadas as amostras de referência com os compósitos contendo partículas de madeira.

Na Figura 4 estão apresentados os resultados de determinação do índice de consistência dos compósitos cimentícios. É possível observar que, para as partículas de compensado e eucalipto, as composições P1 e P3 pouco diferiram, e a maior influência pode ser observada para as partículas $\mathrm{P} 1,2$, que contribuíram para a diminuição do índice de consistência e, consequentemente, para a perda de trabalhabilidade do compósito.

Entretanto, para os compósitos produzidos com as partículas de pínus, os resultados indicam que a diminuição das dimensões das partículas de madeira contribuiu para o aumento do índice de consistência. Isso pode ter ocorrido pelo fato de que, ao produzir compósitos com as partículas com menor dimensão, a massa unitária destas foi maior, produzindo menor volume de madeira na mistura e, consequentemente, menor absorção de água (LI; WANG; WANG, 2006; ANDIÇ-CAKIR et al., 2014; ÇOMAK; BIDECI; BIDECI, 2018). Além disso, o menor tamanho das partículas pode ter propiciado menor atrito entre elas, uma vez que as partículas de madeira de maior dimensão tendem a apresentar um formato mais lamelar.

\section{Figura 3 - Resultado dos tempos de início e fim de pega dos compósitos de cimento-madeira}

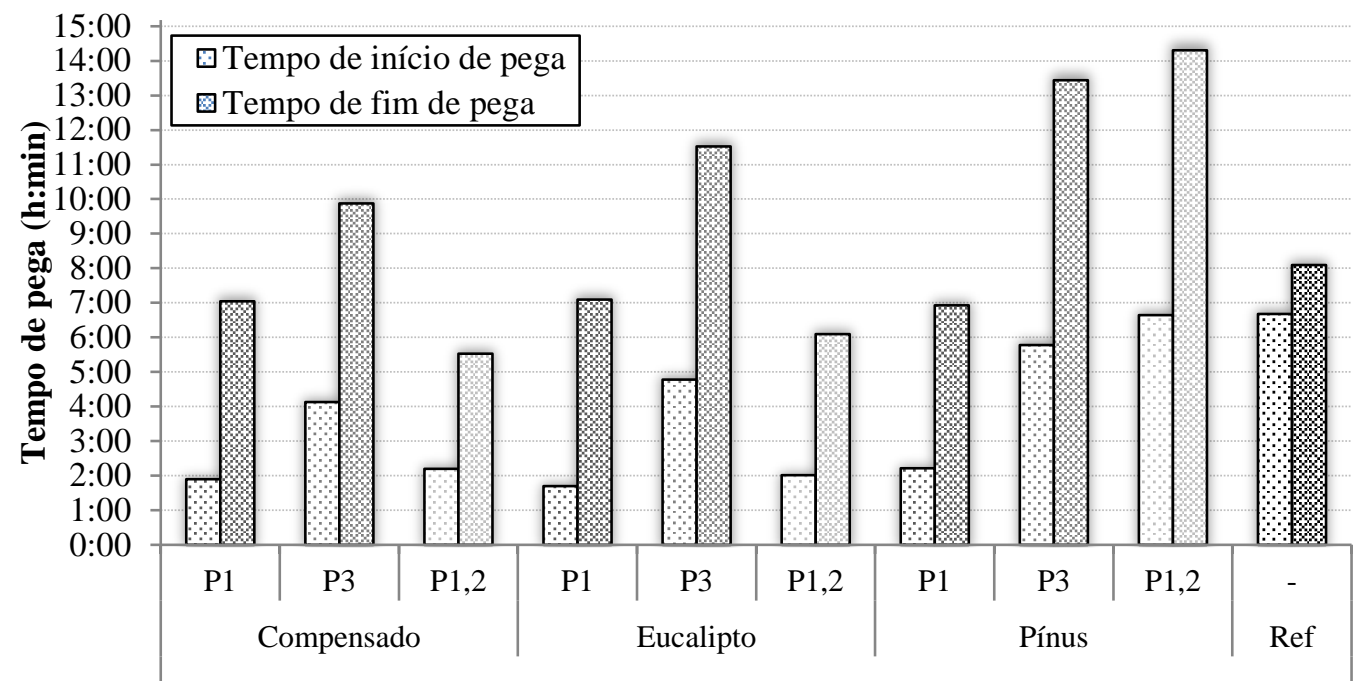


É possível verificar ainda que o maior índice de consistência foi obtido para as amostras de referência, indicando que a presença de partículas de madeira é capaz de contribuir para a diminuição desse parâmetro (MANZUR; AZIZ, 1982; SAVASTANO et al., 1999; ONUAGULUCHI; BANTHIA JUNIOR, 2016).

Os resultados de densidade de massa fresca (Figura 5) indicam que a composição granulométrica das partículas de madeira exerce pouca influência nesse parâmetro, isso porque os compósitos de cimento apresentaram valores entre $1,65 \mathrm{~g} / \mathrm{cm}^{3}$ e $1,70 \mathrm{~g} / \mathrm{cm}^{3}$.
Pesquisadores afirmam que a proporção de madeira na composição e a densidade dessas partículas são consideradas pequenas quando comparadas à proporção e à massa específica do aglomerante utilizado (TURGUT, 2007; MORALES-CONDE; RODRÍGUEZ-LIÑÁN; PEDREÑO-ROJAS, 2016). É possível observar ainda que a maior densidade de massa fresca foi obtida para as amostras de referência, o que indica que a presença de partículas de madeira contribui para a diminuição da densidade dos compósitos (AL RIM et al., 1999; ANDIÇ-ÇAKIR et al., 2014; XIE et al., 2015).

\section{Figura 4 - Resultado do índice de consistência dos compósitos de cimento-madeira}

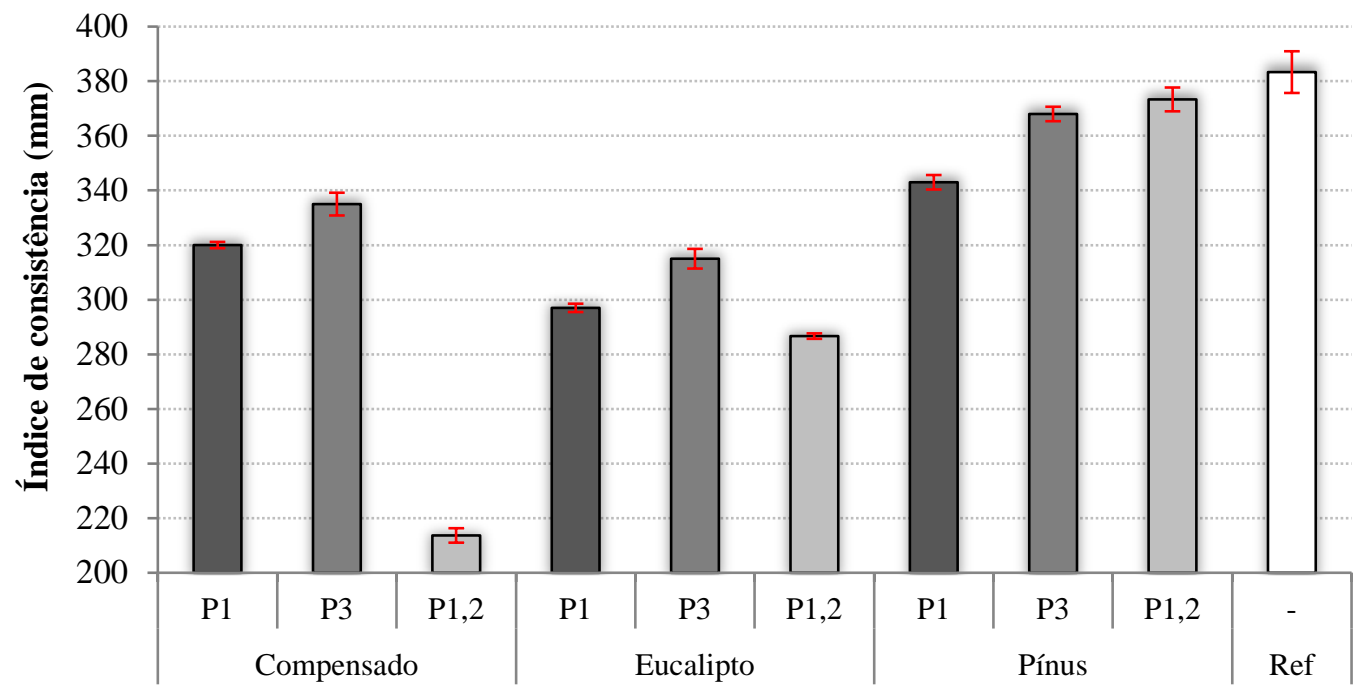

Figura 5 - Resultado da densidade de massa fresca dos compósitos de cimento-madeira

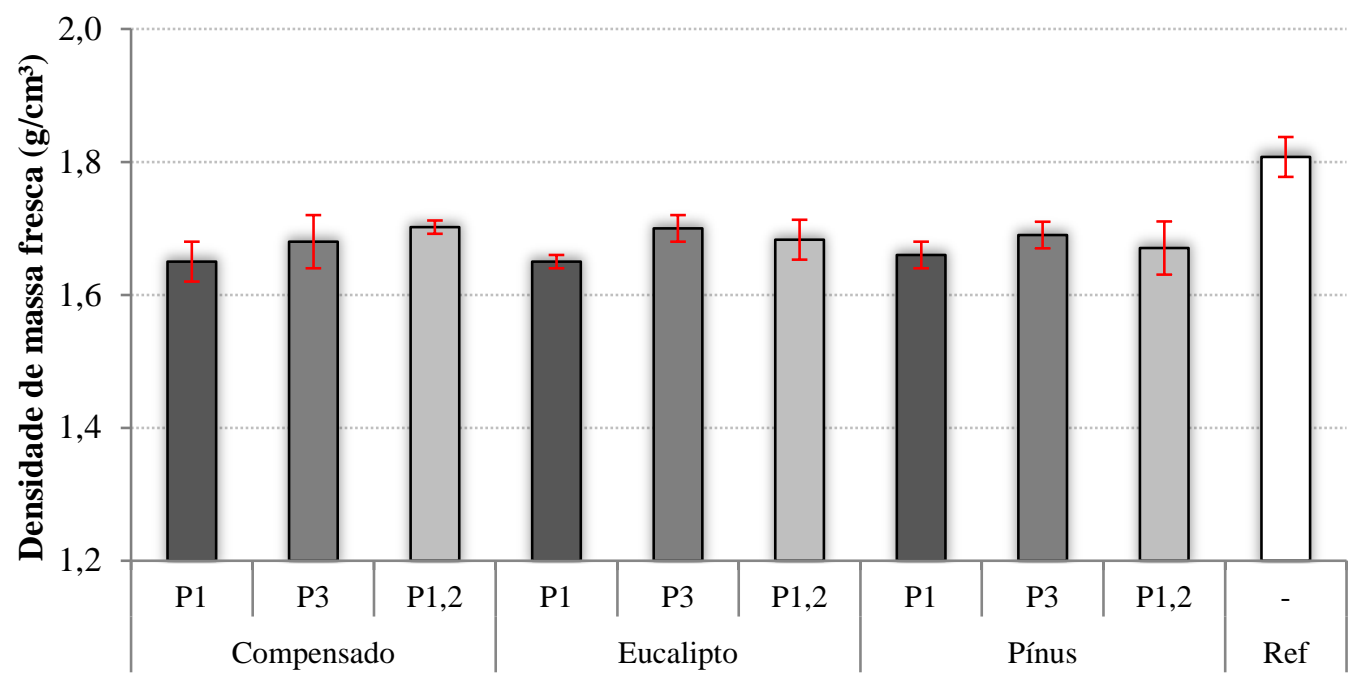




\section{Análise da influência das composições granulométricas das partículas no estado endurecido}

Os resultados de densidade de massa endurecida aos 28 dias dos compósitos de cimento-madeira (Figura 6) evidenciam que os maiores valores foram obtidos para amostras que continham as partículas P3. Isso porque essas partículas, independentemente do tipo de madeira - seja de compensado, de eucalipto ou de pínus -, apresentaram os maiores valores de massa unitária seca. Ao realizar a dosagem dos materiais em massa, as partículas com menor massa unitária passam a apresentar menor volume na mistura, o que contribui para o aumento da densidade (TURGUT, 2007; MORALES-CONDE; RODRÍGUEZ-LIÑÁN; PEDREÑO-ROJAS, 2016).

O mesmo ocorre com as partículas $\mathrm{P} 1,2$, cujos valores de massa unitária foram os menores obtidos entre as composições granulométricas avaliadas das partículas e, consequentemente, apresentaram os menores valores de densidade de massa endurecida. Isso ocorre porque, devido ao maior volume de partículas de madeira, menor tende a ser a densidade da composição (TURGUT, 2007; MORALES-CONDE; RODRÍGUEZ-LIÑÁN; PEDREÑO-ROJAS, 2016).

A análise estatística indicou ainda que existe diferença estatística entre os compósitos ao considerar os três fatores avaliados - a composição granulométrica da partícula (P1, P3 e P1,2), o tipo de madeira (compensado, eucalipto e pínus) e a proporção de madeira na composição (7,5\%, 11\% e $15 \%$ ), ou seja, são capazes de influenciar a densidade de massa endurecida dos compósitos. De modo geral, verificou-se que os compósitos de cimento-madeira produzidos com a composição P1,2 ou com pínus ou com a proporção de $15 \%$ tendem a apresentar os menores valores de densidade de massa endurecida.

Por fim, observou-se que as densidades de massa endurecida dos compósitos contendo partículas de madeira foram significativamente inferiores aos resultados obtidos para as amostras de referência.

Os resultados de resistência à tração na flexão aos 28 dias dos compósitos de cimento-madeira estão apresentados na Figura 7. É possível observar diferentes comportamentos de acordo com o tipo de madeira (compensado, eucalipto e pínus) presente na composição.

Nos compósitos produzidos com as partículas de compensado, verificou-se que as menores resistências foram obtidas para os compósitos contendo as partículas P3 devido ao menor volume de partículas na mistura, conforme discutido anteriormente. Para os compósitos produzidos com as partículas de eucalipto, os resultados apresentaram-se muito próximos, indicando pouca influência da composição granulométrica das partículas e da proporção de madeira na composição.

\section{Figura 6 - Resultado da densidade de massa endurecida dos compósitos de cimento-madeira}

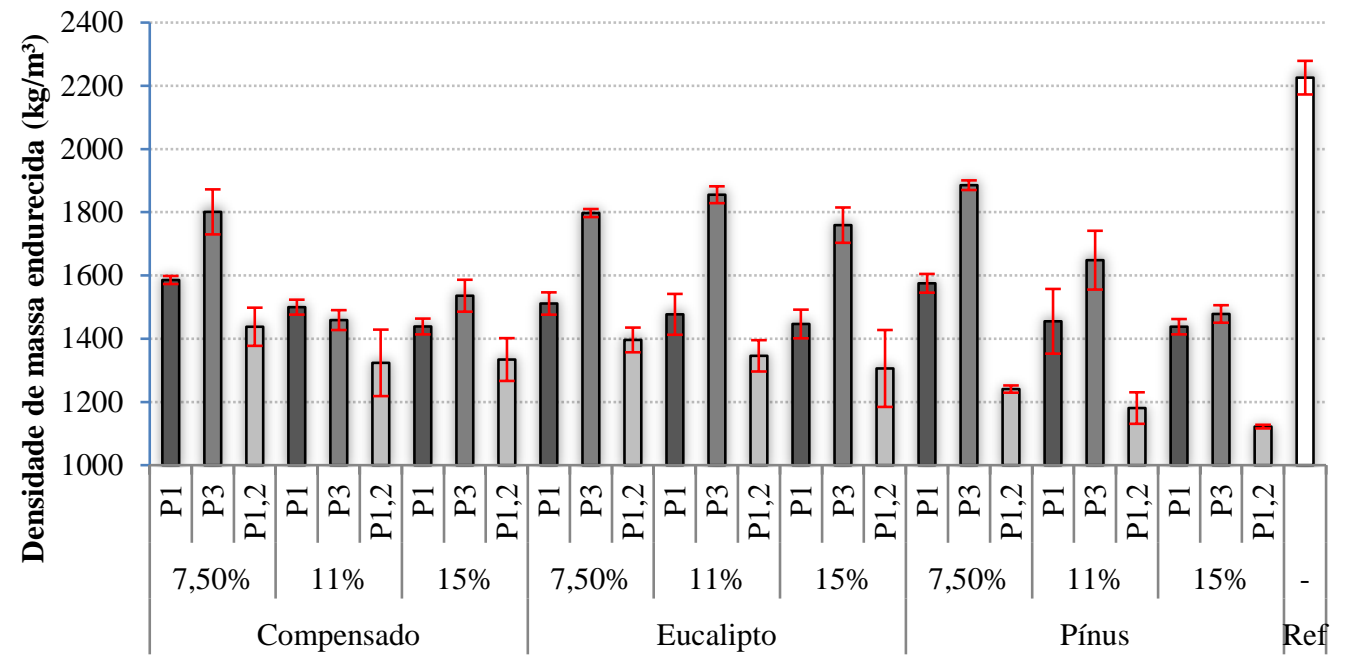


Diferentemente desses, os compósitos de cimento produzidos com as partículas de pínus apresentaram diminuição da resistência conforme a diminuição da dimensão das partículas, observada a partir de sua composição granulométrica. Isso pode ter sido provocado pela maior liberação de extrativos pelas partículas de madeira, que contribuem para uma maior incompatibilidade entre o cimento Portland e a espécie em questão - conforme observado no estado fresco através do ensaio de tempo de pega , resultando na diminuição da resistência. Assim como observado por Onuaguluchi e Banthia (2016), a influência das fibras naturais no desempenho mecânico dos compósitos ainda é um limitante para a propagação de seu uso.

A análise estatística indica ainda que, ao considerar como fator as composições granulométricas das partículas, foi obtida equivalência estatística dos compósitos produzidos com P3 e P1,2. Em outras palavras, para os compósitos produzidos com as partículas P3 e P1,2, os resultados de resistência à tração na flexão podem ser considerados iguais entre si. E, ao considerar como fator a proporção de madeira na composição $(7,5 \%, 11 \%$ e $15 \%)$, foi obtida equivalência estatística entre os teores 7,5\% e $15 \%$. Foi observada diferença estatística ao considerar como fator o tipo de madeira (compensado, eucalipto e pínus) porque, assim como observado por Tonoli et al. (2010), as partículas de eucalipto tendem a apresentar melhor dispersão e melhor ligação com a matriz cimentícia quando comparadas às partículas de pínus, o que contribui para a melhoria do desempenho mecânico.

Dessa maneira, os compósitos produzidos com a composição granulométrica P1 ou com as partículas de compensado ou com a proporção de $11 \%$ tendem a apresentar maior resistência à tração na flexão.
Vale destacar ainda que os compósitos de cimentomadeira produzidos com as partículas de compensado e eucalipto nas composições granulométricas P3 e P1,2 apresentaram resultados de resistência à tração na flexão iguais ou superiores às amostras de referência.

Os resultados de resistência à compressão aos 28 dias dos compósitos de cimento-madeira estão apresentados na Figura 8. É possível observar diferentes comportamentos de acordo com o tipo de madeira (compensado, eucalipto e pínus), como descrito para os resultados de resistência à tração na flexão.

Assim como observado para a resistência à tração na flexão para os compósitos produzidos com as partículas de compensado, verificou-se que as menores resistências foram obtidas para os compósitos contendo as partículas P3. Enquanto que, com as partículas de eucalipto, os resultados indicaram pouca influência das composições granulométricas das partículas e da proporção de madeira na composição. Diferentemente destes, os compósitos de cimento produzidos com as partículas de pínus apresentaram diminuição da resistência conforme a diminuição da dimensão das partículas (AL RIM et al., 1999; TURGUT, 2007), conforme explicado anteriormente.

A análise estatística indica ainda que para os fatores avaliados (composição granulométrica das partículas, tipo e proporção de madeira) foi observada diferença estatística. De modo geral, verificou-se que os compósitos de cimento-madeira produzidos com a composição P1,2 ou com compensado ou com a proporção de 7,5\% tendem a apresentar os maiores valores de resistência à compressão.

\section{Figura 7 - Resultado da resistência à tração na flexão dos compósitos de cimento-madeira}

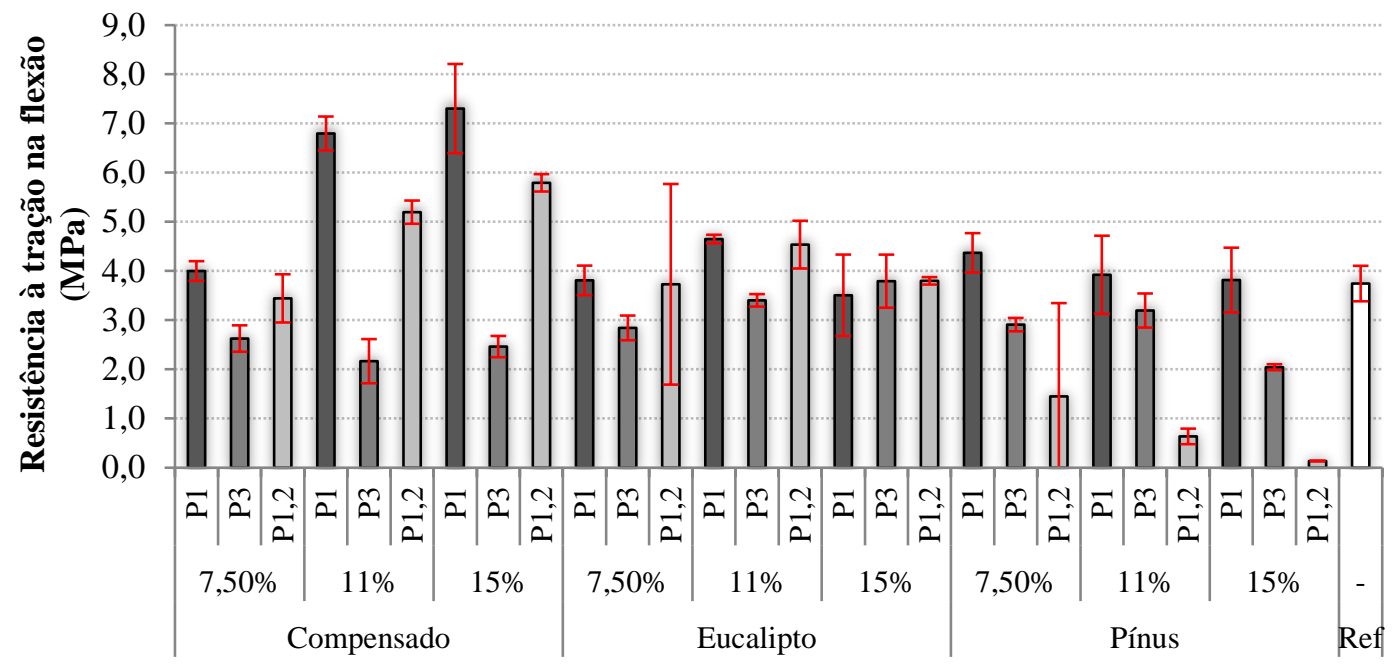


Figura 8 - Resultado da resistência à compressão dos compósitos de cimento-madeira

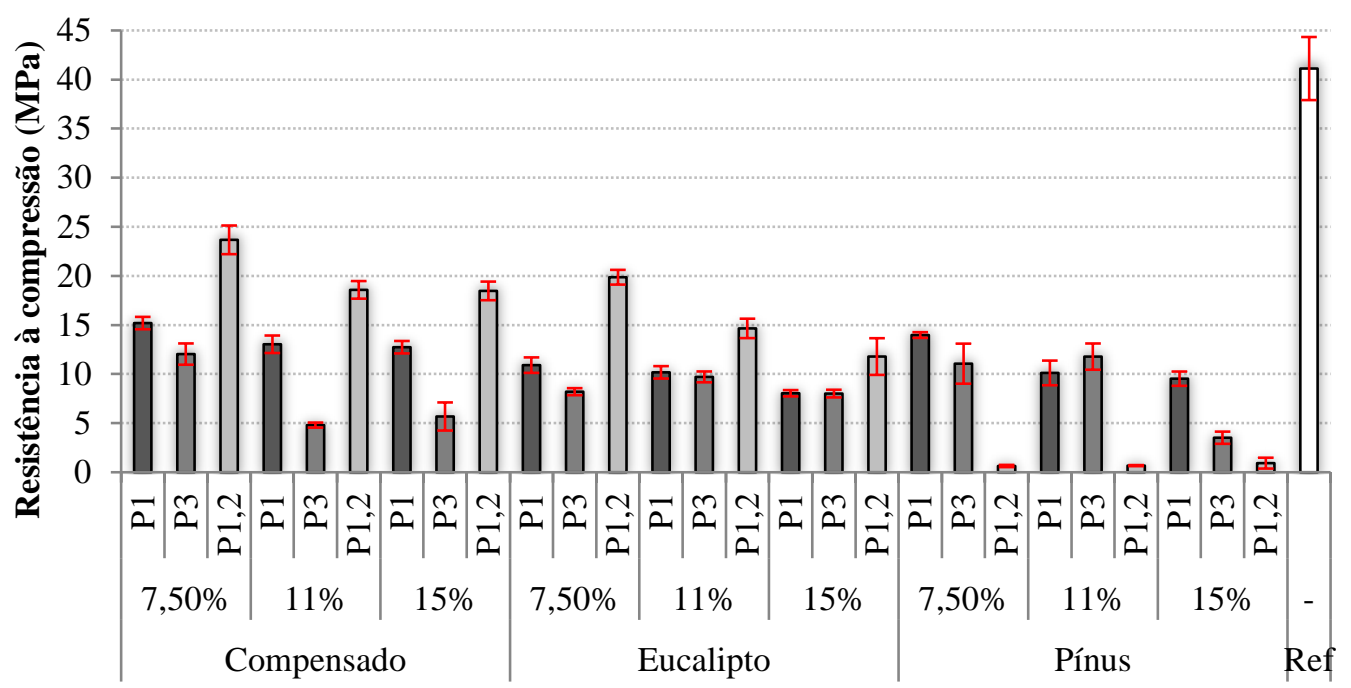

Por fim, observou-se que as resistências à compressão dos compósitos contendo partículas de madeira foram significativamente inferiores aos resultados obtidos para as amostras de referência.

\section{Conclusões}

Neste artigo foi verificada a influência das composições granulométricas das partículas de resíduos de madeira nas propriedades de compósitos cimentícios. Foi observado ainda o efeito de três diferentes tipos de madeira (compensado, eucalipto e pínus) e de três diferentes proporções de madeira na composição $(7,5 \%, 11 \%$ e 15\%). Com base nos resultados, as seguintes conclusões foram obtidas.

(a) os resultados de massa unitária seca das partículas indicam que, para dosagens realizadas em massa, ocorre diminuição no volume de madeira presente nos traços produzidos com as partículas moídas três vezes (P3) quando comparada às partículas moídas apenas uma vez (P1) e as partículas passantes na peneira 1,2 mm $(\mathrm{P} 1,2)$;

(b) o aditivo acelerador de pega utilizado mostrou-se eficiente para diminuir o tempo de início de pega dos compósitos produzidos com partículas de madeira quando comparados às amostras de referência;

(c) os compósitos de cimento-madeira produzidos com partículas de pínus apresentaram maior influência na composição granulométrica P1,2, tanto para os tempos de pega como para o índice de consistência; (d) observou-se que o maior índice de consistência foi obtido para as amostras de referência, indicando que a presença de partículas de madeira é capaz de contribuir para sua diminuição. Além disso, as composições P1,2 de compensado e de eucalipto contribuíram em maior magnitude para a perda de trabalhabilidade dos compósitos de cimento-madeira.;

(e) as maiores densidades de massa endurecida foram obtidas para amostras que continham as composições P3. Isso porque essas partículas, independentemente do tipo de madeira (seja de compensado, de eucalipto ou de pínus), apresentaram os maiores valores de massa unitária seca e, consequentemente, menor volume de partículas na composição. Em contrapartida, os compósitos de cimento-madeira produzidos com as composições $\mathrm{P} 1,2$ ou com o pínus ou com a proporção de $15 \%$ tendem a apresentar os menores valores de densidade de massa endurecida. Todos os compósitos contendo partículas de madeira apresentaram densidade de massa endurecida inferior às amostras de referência;

(f) os compósitos de cimento-madeira produzidos com as partículas de compensado e de eucalipto nas composições granulométricas P1 e P1,2 apresentaram resultados de resistência à tração na flexão iguais ou superiores às amostras de referência, diferentemente dos resultados de resistência à compressão, em que os resultados dos compósitos contendo partículas de madeira foram significativamente inferiores aos resultados obtidos com as amostras de referência; e

(g) os resultados de resistência apresentaram diferentes comportamentos, principalmente de acordo com o tipo de madeira (de compensado, de 
eucalipto ou de pínus) utilizado para a produção dos compósitos. De modo geral, os compósitos produzidos com a composição granulométrica $\mathrm{P} 1$ ou com as partículas de compensado ou com a proporção de $11 \%$ tendem a apresentar maior resistência à tração na flexão quando comparados aos demais compósitos produzidos neste estudo, enquanto os compósitos de cimento-madeira produzidos com as composições $\mathrm{P} 1,2$ ou com compensado ou com a proporção de 7,5\% tendem a apresentar os maiores valores de resistência à compressão.

\section{Referências}

ADEDEJI, Y. M. D.; AJAYI, B. Cost-Effective Composite Building Panels for Walls and Ceilings in Nigeria. In: INTERNATIONAL INORGANICBONDED FIBER COMPOSITES

CONFERENCE, 11., Madrid, 2008.

Proceedings... Madrid, 2008.

AKIL, H. M. et al. Kenaf Fiber Reinforced Composites: a review. Materials and Design, v. 32, n. 8-9, p. 4107-4121, 2011.

AL RIM, K. et al. Influence of the Proportion of Wood on the Thermal and Mechanical

Performances of Clay-Cement-Wood Composites.

Cement and Concrete Composites, v. 21, p. 269276, 1999.

AMERICAN SOCIETY FOR TESTING AND MATERIALS. C-136: standard test method for sieve analysis of fine and coarse aggregates. West Conshohocken, 2014a.

\section{AMERICAN SOCIETY FOR TESTING AND}

MATERIALS. C-1437: standard test method for flow of hydraulic cement mortar. West

Conshohocken, 2015.

AMERICAN SOCIETY FOR TESTING AND MATERIALS. C-270: standard specification for mortar for unit masonry. West Conshohocken, 2014d.

AMERICAN SOCIETY FOR TESTING AND MATERIALS. C-29: standard test method for bulk density (unit weight) and voids in aggregate. West Conshohocken, 2017.

AMERICAN SOCIETY FOR TESTING AND MATERIALS. C-308: standard test methods for working, initial setting, and service strength setting times of chemical-resistant resin mortars. West Conshohocken, 2012.

AMERICAN SOCIETY FOR TESTING AND MATERIALS. C-348: standard test method for flexural strength of hydraulic-cement mortars. West Conshohocken, 2014b.
AMERICAN SOCIETY FOR TESTING AND MATERIALS. C-349: standard test method for compressive strength of hydraulic-cement mortars (using portions of prisms broken in flexure). West Conshohocken, 2014c.

AMERICAN SOCIETY FOR TESTING AND MATERIALS. D-4442: standard test methods for direct moisture content measurement of wood and wood-based materials. West Conshohocken, 2016.

ANDIÇ-ÇAKIR, O. et al. Physical and Mechanical Properties of Randomly Oriented Coir Fiber-Cementitious Composites. Composites Part B: Engineering, v. 61, p. 49-54, 2014.

ASHOUR, T. et al. Thermal Conductivity of Unfired Earth Bricks Reinforcedby Agricultural Wastes with Cement and Gypsum. Energy and Buildings, v. 104, p. 139-146, 2015.

BOUGUERRA, A. et al. Water Sorptivity and Pore Structure of Wood Cementitious Composites. Magazine Concrete Research, v. 54, v. 2, p. 103112, 2002.

ÇOMAK, B.; BIDECI, A.; BIDECI, O. S. Effects of Hemp Fibers on Characteristics of Cement Based Mortar. Construction and Building Materials, v. 169, p. 794-799, 2018.

CORINALDESI, V.; MAZZOLI, A.; SIDDIQUE, R. Characterization of Lightweight Mortars Containing Wood Processing By-Products Waste. Construction and Building Materials, v. 123, p. 281-289, 2016.

EUROPEAN COMMISSION. Resource Efficient Use of Mixed Wastes Improving Management of Construction and Demolition Waste - Final report. Brussels, 2017.

HUANG, W.-L. et al. Recycling of Construction and Demolition Waste Via a Mechanical Sorting Process. Resources, Conservation and Recycling, v. 37, p. 23-37, 2002.

HULL, D.; CLYNE, T. W. An Introduction to Composite Materials. $2^{\text {nd }}$. ed. Cambridge: Cambridge University Press, 1996.

KARADE, S. R.; IRLE, M.; MAHER, K. Influence of Granule Properties and Concentration on Cork-Cement Compatibility. Holz als Roh-und Werkstoff, v. 64, n. 4, p. 281-286, 2006.

KELLY, A. (Ed.). An Introduction to Composite Materials. In: CONCISE Encyclopedia of Composite Materials. Oxonia: Pergamon Press, 1994. 
KHORAMI, M.; GANJIAN, E. The Effect of Limestone Powder, Silica Fume and Fibre Content on Flexural Behaviour of Cement Composite Reinforced by Waste Kraft Pulp. Construction and Building Materials, v. 46, p. 142-149, 2013.

\section{LATORRACA, J. V. F. Eucalyptus spp na Produção de Painéis de Cimento-Madeira. Curitiba, 2000. $191 \mathrm{f}$. Tese (Doutorado em Engenharia Florestal) - Setor de Ciências Agrárias, Universidade Federal do Paraná, Curitiba, 2000.}

LATORRACA, J. V.; IWAKIRI, S.; LELIS, R. C. Efeito Inibidor de Cinco Espécies Florestais Sobre a Cura do Compósito Cimento-Madeira. Floresta e Ambiente, v. 6, n. 1, p. 76-82, 1999.

LI, Z. J.; WANG, L. J.; WANG, X. G. Flexural Characteristics of Coir Fiber Reinforced Cementitious Composites. Fibers and Polymers, v. 7, p. 286-294, 2006.

LIMA, A. J. M. Utilização de Resíduo de Pínus spp e Materiais Cimentícios Alternativos na Produção de Blocos Para Alvenaria Estrutural. Curitiba, 2009. $281 \mathrm{f}$. Tese (Doutorado em Engenharia Florestal) - Setor de Ciências Agrárias, Universidade Federal do Paraná, Curitiba, 2009.

LIMA, A. J. M.; IWAKIRI, S. Produtos Alternativos na Produção de Blocos para Alvenaria Estrutural. Floresta e Ambiente, v. 18, n. 3, p. 310-323, 2011.

MANSUR, M. A.; AZIZ, M. A. A Study on Jute Fibre Reinforced Cement Composites. The International Journal of Cement Composites and Lightweight Concrete, v. 4, n. 2, p. 75-82, 1982.

MATHUR, V. K. Composite Materials from Local Resources. Construction and Building

Materials, v. 20, p. 470-477, 2006.

MILLER, S. A.; LEPECH, M. D.; BILLINGTON, S. L. Application of Multi-Criteria Material Selection Techniques to Constituent Refinement in Biobased Composites. Materials and Design, v. 52, p. 1043-1051, 2013.

MORALES-CONDE, M. J.; RODRÍGUEZLIÑÁN, C.; PEDREÑO-ROJAS, M. A. Physical and Mechanical Properties of Wood-Gypsum Composites from Demolition Material in Rehabilitation Works. Construction and Building Materials, v. 114, p. 6-14, 2016.

MOSLEMI A. A.; GARCIA, J. F.; HOFSTRAND, A. D. Effect of Various Treatments and Additives on Wood-Portland Cement Water Systems. Wood and Fiber Science, v. 15, n. 2, p. 164-176, 1983.
NA, B. et al. Wood-Cement Compatibility Review. Wood Research, v. 59, n. 5, p. 813-825, 2014.

ONUAGULUCHI, O.; BANTHIA, N. Plant-Based Natural Fibre Reinforced Cement Composites: a review. Cement and Concrete Composites, v. 68, p. 96-108, 2016.

\section{PARCHEN, C. F. A. Compósito Madeira} Cimento de Baixa Densidade Produzido com Método de Compactação Vibro Dinâmica. Curitiba, 2012. 172 f. Tese (Doutorado em Engenharia Florestal) - Setor de Ciências Agrárias, Universidade Federal do Paraná, Curitiba, 2012.

PEDREÑO-ROJAS, M. A. et al. Eco-Efficient Acoustic and Thermal Conditioning Using False Ceiling Plates Made From Plaster and Wood Waste. Journal of Cleaner Production, v. 166, p. 690-705, 2017.

PERVAIZ, M.; SAIN, M. M. Carbon Storage Potential in Natural Fiber Composites. Resources, Conservation and Recycling, v. 39, p. 325-340, 2003.

PIETRINI, M. et al. Comparative Life Cycle Studies on Poly(3- hydroxybutyrate)-Based Composites as Potential Replacement For Conventional Petrochemical Plastics.

Biomacromolecules, v. 8, n. 7, p. 2210-2218, 2007.

RAMAKRISHNA, G.; SUNDARARAJAN, T. Impact Strength of a Few Natural Fibre Reinforced Cement Mortar Slabs: a comparative study.

Cement and Concrete Composites, v. 27, p. 547553, 2005.

\section{ROCHA, T. M. S. Contribuição ao}

Desenvolvimento de Placas Para Vedação Vertical Produzidas Com Resíduo de Madeira de Construção, Utilizando Gesso e Cimento Como Aglomerante. Curitiba, 2017. 204 f. Dissertação (Mestrado em Engenharia Civil) Universidade Federal do Paraná, Curitiba, 2017.

SAVASTANO JUNIOR, H. et al. Fracture and Fatigue of Natural Fiber Reinforced Cementitious Composites. Cement and Concrete Composites, v. 31, p. 232-243, 2009.

SAVASTANO JUNIOR, H. et al. Plant Fibre Reinforced Cement Components for Roofing. Construction and Building Materials, v. 13, p. 433-438, 1999.

SELLAMI, A.; MERZOUD, M.; AMZIANE, S. Improvement of Mechanical Properties of Green Concrete by Treatment of the Vegetals Fibers. Construction and Building Materials, v. 47, p. 1117-1124, 2013. 
SIMATUPANG, M. H. Der Wasserbedarf bei der Herstellung zementgebunder Holzspanplatten. Holz als Roh-und Werkstoff, v. 27, p. 379-382, 1979.

\section{SMITH, E. H. Mechanical Engineer's Reference} Book. Oxonia: Butterworth-Heinemann Ltd, 1994.

TOLEDO FILHO, R. D. et al. Free, Restrained and Drying Shrinkage of Cement Mortar Composites Reinforced With Vegetable Fibres. Cement and Concrete Composites, v. 27, p. 537546, 2005.

TONOLI, G. H. D. et al. Eucalyptus Pulp Fibres as Alternative Reinforcement to Engineered CementBased Composites. Industrial Crops and Products, v. 31, n. 2, p. 225-232, 2010.

TONOLI, G. H. D. et al. Processing and Dimensional Changes of Cement Based Composites Reinforced with Surface-Treated Cellulose Fibres. Cement and Concrete Composites, v. 37, p. 68-75, 2013.

TURGUT, P. Cement Composites with Limestone Dust and Different Grades of Wood Sawdust. Building and Environment, v. 42, p. 3801-3807, 2007.
VÄNTSI, O.; KÄRKI, T. Environmental Assessment of Recycled Mineral Wool and Polypropylene Utilized in Wood Polymer Composites. Resources, Conservation and Recycling, v. 104, p. 38-48, 2015.

XIE, X. et al. Cellulosic Fibers from Rice Straw and Bamboo Used as Reinforcement of CementBased Composites for Remarkably Improving Mechanical Properties. Composites Part B: Engineering, v. 78, p. 153-161, 2015.

XU, X. et al. Life Cycle Assessment of WoodFibre-Reinforced Polypropylene Composites. Journal of Materials Processing Technology, v. 198, n. 1-3, p. 168-177, 2008.

YOUNGQUIST, J. A. Wood Handbook: wood as an engineering material: wood-based composites and panel products. Madison: Forest Products Laboratory, 1999.

YUAN, H.; SHEN, L. Trend of the Research on Construction and Demolition Waste Management. Waste Management, v. 31, p. 670-679, 2011.

Thaísa Mariana Santiago Rocha

Programa de Pós-Graduação em Engenharia de Construção Civil | Universidade Federal do Paraná | Centro Politécnico, Jardim das Américas | Curitiba - PR - Brasil | CEP 80035-210| Tel.: (41) 99901-5141 | E-mail: thaisamsrocha@yahoo.com.br

Leonardo Fagundes Rosemback Miranda

Departamento de Construção Civil | Universidade Federal do Paraná | Tel.: (41) 3361-3364 | E-mail: reciclagem.miranda@gmail.com

\section{Carlos Frederico Alice Parchen}

Departamento de Construção Civil, Setor de Tecnologia | Universidade Federal do Paraná | Campus IV, Jardim das Américas | Curitiba PR - Brasil | Caixa Postal 19011 | CEP 81531-980 | Tel.: (41) 3361-3000 Ramal 3001| E-mail: parchen.ufpr@gmail.com

Revista Ambiente Construído

Associação Nacional de Tecnologia do Ambiente Construído

Av. Osvaldo Aranha, 99 - 3o andar, Centro

Porto Alegre - RS - Brasil

CEP $90035-190$

Telefone: +55 (51) 3308-4084

Fax: +55 (51) 3308-4054

www. seer. ufrgs. br/ ambienteconstruido

E-mail: ambienteconstruido@ufrgs.br 